\title{
Medical Informatics : On an ongoing road to the final destination
}

\author{
Mohammad Babaei \\ Cancer Institute and Research Centre \\ University of Medical Sciences of Tehran \\ Iran
}

\section{Maryam Ganjalikhani}

Kerman Research Center

University of Medical Sciences of Kerman

Iran

Sri Lanka Journal of Bio-Medical Informatics 2010;1(3):186-190

DOI: 10.4038/sljbmi.v1i3.1985

\begin{abstract}
Medical informatics is the intersection of information science, computer science, and health care. It expedites medicine to support clinical practice, decision-making and research. Health informatics or medical informatics is the acceptable specialty to improve health care by using medical data. It seems as if it is an essential part of medical practice as it can decrease medical errors. Informatics has emerged to fill the space between physicians and IT services. So now and in future medicine owes many of progresses to medical informatics. Learning medical informatics is useful and necessary to physicians. There are some problems within the context; opponents use these as a parapet to protect themselves. Here, the authors bring an example: electronic medical data includes a wide range of data relating to a patient's health, including details of his/her past, current and future diagnoses, treatments and medication. Inaccuracies in a patient's medical data can result in misdiagnosis and medical professionals can face legal sanctions for malpractice due to inaccurate data; disclosure of medical data can result in prejudicial treatment and researchers can be criminally liable for unauthorized disclosure of medical data. Also patient data is private and must be protected. As Medical informatics decreases medical errors, helps physician to increase the quality of their workout, learning and using this science is necessary for every physician this concept should be assigned important especially in developing countries.
\end{abstract}

Keywords: Medical informatics, Health informatics, Learning

Sri Lanka Journal of Bio-Medical Informatics 2010;1(3):186-190

DOI: $10.4038 /$ sljbmi.v1i3.1985

\section{Introduction}

If physiology literally means 'the logic of life', and pathology is 'the logic of disease', then health informatics is the logic of healthcare ${ }^{(1)}$. The question which comes to mind is that: what is medical informatics? In fact, Health informatics, Healthcare informatics or Medical informatics, is the intersection of information science, computer science, and health care. Medical informatics develops and assesses methods and systems for the acquisition, processing, and interpretation of patient data with the help of knowledge that is obtained in scientific research. 
NHS Faculty of Health Informatics defines medical informatics as "the knowledge, skills and tools which enable information to be collected, managed, used and shared to support the delivery of healthcare and promote health." The latest edition of Shortliffe's textbook defines the discipline as "a field of study concerned with the broad issues in the management and use of biomedical information, including medical computing and the study of the nature of medical information" (2). Medical informatics is the discipline concerned with the systematic processing of data, information and knowledge in medicine and health care. The ultimate goal should always be to improve the quality of health care. Overall, it is the science of information management in healthcare, and its application to support clinical practice, decision-making and research.

\section{Domains of medical informatics}

Medical informatics began to take off in the US in the 1950s with the advent of the computer. Health informatics is the appropriate and innovative application of the concepts and technologies of the information age to improve health care and health ${ }^{(3)}$. The focus on the use of existing and new technologies to facilitate advances in medical imaging and medical informatics is often directed to the technical capabilities and possibilities that these technologies bring, so medical professionals turn to medical informatics as an essential element of medical practice to reduce errors and to teach and diagnose better.

The word informatics increasingly is used as an adjective to sell the latest health-care technology. Vendors of clinical Information systems hope to impress customers by claiming that their products are based on "informatics techniques" and "informatics methods." The report of the Institute of Medicine on the problem of medical errors specifically identified information technology as a large element of the needed solution ${ }^{(5)}$.

Although the name 'health informatics' only came into use around 1973, it is a discipline that is as old as healthcare itself. It was born the day a clinician first wrote down some impressions about a patient's illness, and used these to learn how to treat the next patient. With the evolution of the field, health informatics is probably best defined in the context of eHealth, which is generally accepted as a term composed of two elements:

- Health informatics (related to the collection, analysis and movement of health information and data to support health care), and

- Telehealth (related to direct, e.g. videoconferencing, or indirect, e.g. website delivery of health information or health care to a recipient).

eHealth encompasses products, systems and services, including tools for health authorities and professionals, as well as personalized health systems for patients and citizens. The scope of eHealth includes desktop to bedside to population health activities, which present complex information management challenges to support individualized patient care $^{(6)}$.

A useful model of the emergence of informatics is to consider the different roles of clinicians, management and IT services in health care. Each group has its own perspective and frequently pulls in different directions. Informatics has emerged to fill the space.

Health informatics is thus as much about computers as cardiology is about stethoscopes. Rather than drugs, X-ray machines or surgical instruments, the tools of informatics are more 
likely to be clinical guidelines, formal health languages, information systems, or communication systems like the Internet. These tools, however, are only a means to an end, which is the delivery of the best possible healthcare ${ }^{(7)}$.

The progress of information processing and information technology changes the world. 'Transforming healthcare through innovative use of information technology for the $21 \mathrm{st}$ century' was the topic of the 6th International Conference on Health and Medical Informatics Education and a special issue of the International Journal of Medical Informatics. Today health professionals who are well-educated in health informatics or medical informatics are needed. Raising the scope and the quality of education in the field of health and medical informatics would help to raise the quality and efficiency of healthcare ${ }^{(8)}$, so educating general practitioners, post graduates, and specialists in this field seems to be useful and may be necessary. Education in this field is for providing learners with sound basic medical background in order to be able to understand and reflect on medical problems to be solved ${ }^{(9)}$.

\section{Opponents against medical informatics : What do they say?}

It is usually easier to criticize and censure. Naturally medical informatics also has opponents to some aspects of it. But what is the main concept of defiance? These are concerns ranging from ethics to data security. It seems as if the borders and domains of medical informatics are vague, some even still don't dare to call it a science ${ }^{(10,11,12)}$.

The benefits of utilizing computer technology in medical practice has long been felt, and the risks associated with using this technology has also been evident to some extent ${ }^{(13)}$. Medical informatics systems have the ultimate goal of improving the quality of health care. However, these systems have also the potential to compromise the quality of health care if they are misused, intrinsically faulty or entail unexpected side effects ${ }^{(14)}$. Medical informatics has the noble goal to improve the quality of health care and provide service to those who suffer, but good intentions are not enough.

Electronic medical data includes a wide range of data relating to a patient's health, including details of his/her past, current and future diagnoses, treatments and medication ${ }^{(15)}$. Electronic medical data also includes electronic/digital images such as an X-ray or mammography. Data processing includes collection, storage, organization, retrieval, use, dissemination, consultation and transmission among others. The processing of this data is critical since inaccuracies and misuse can have detrimental consequences for patients, medical professionals and researchers. Inaccuracies in a patient's medical data can result in misdiagnosis and medical professionals can face legal sanctions for malpractice due to inaccurate data; disclosure of medical data can result in prejudicial treatment and researchers can be criminally liable for unauthorized disclosure of medical data ${ }^{(16)}$.

The proliferation of electronic medical data has undoubtedly resulted in significant benefits (e.g. enhanced patient autonomy, better clinical treatment, and advances in health research), however, there are also important legal challenges such as: the privacy of health information; reliability and quality of health data; and tort-base liability. These are also interconnected because the degree of privacy protection determines the reliability and quality of the data, which in turn determines tortbased liability for acts such as malpractice (e.g. due to inaccurate data) or privacy invasions (e.g. unauthorized access, modification or disclosure) ${ }^{(4)}$. 


\section{Conclusion}

Medical informatics develops and assesses methods and systems for the acquisition, processing, and interpretation of patient data with the help of knowledge that is obtained in scientific research.

Medical informatics nowadays helps clinicians in areas such as Electronic Health Record, Medical Imaging Processing, Information Retrieval Systems and research. We think that medical informatics education is undoubtedly useful to nurses, physician and all clinicians. By education we mean becoming familiar and using medical informatics, we do not mean that every one become a medical informatician. If education of medical informatics is not available in a country, no one would use it. Every country should pay attention to this science, especially developing countries.

\section{References:}

1. Coiera.E, Guide to Health Informatics 2nd Edition

2. $\quad$ Shortliffe, E.H., Perreault, L.E., Wiederhold, G., and Fagan, L.M. (eds.). MedicalInformatics: Computer Applications in Health Care and Biomedicine, 2nd Edition. NewYork: Springer-Verlag, 2001.

3. Australian College of Health Informatics. Available at achi.org.au/informatics/faq.html.

4. J.G. Hodge, L. Gostin and P. Jacobson, Legal issues concerning Electronic Health Information, JAMA 282 (1999), 1466-71

5. Kohn, L.T., Corrigan, J.M., and Donaldson, M.S. (eds.). To Err Is Human: Building a Safer Health System. Washington: National Academy Press, 2000.

6. The RACGP Curriculum for Australian General Practice, available at www.racgp.org.au/curriculum

7. Charles P. Friedman, and Ume L. Abbas, Is medical informatics a mature science? A review of measurement practice in outcome studies of clinical systems, International Journal of Medical Informatics. 2003;69(2-3):261-72

8. Reinhold Haux, Health and medical informatics education: perspectives for the next decade,International Journal of Medical Informatics, 1998;50(1-3):7-19

9. F.J Leven ${ }^{\mathrm{a}}$, and R Haux, Twenty five years of medical informatics education at Heidelberg/Heilbronn: discussion of a specialized curriculum for medical informatics, International Journal of Medical Informatics, June 1998;50(1-3):31-42

10. E Coiera, SB Dowton, Re-inventing Ourselves - How innovations such as on-line 'just-in-time' CME may help bring about a genuinely evidence-based clinical practice, Medical Journal of Australia, 2000;173:343-44, 
11. E.H. Shortliffe and L.E. Perreault, Editors, Medical Informatics: Computer Applications in Health Care and Biomedicine, Springer, New York (2001).

12. J.G. van Bemmel and M.A. Musen. Handbook of Medical Informatics, Bohn, Stafleu, Van Loghum, Houten, The Netherlands (1997).

13. H. Muller, C. Lovis and A. Geissbuhler, The medGIFT project on medical image retrieval, Proceedings of First International Conference on Medical Imaging and Telemedicine (MIT 2005), August 16 - 19 Wuyi Mountain, China (2005).

14. Günther Gell, Side effects and responsibility of medical informatics, International Journal of Medical Informatics. 2001;64(2-3):69-81

15. W. Lowrance, Privacy and Health Research (1997). Available at http://aspe.os.dhhs.gov/datacncl/PHR.htm

16. Duquenoy.P, George.D and Solomonides.A, Considering something 'ELSE': Ethical, legal and socio-economic factors in medical imaging and medical informatics, Computer Methods and Programs in Biomedicine. December 2008;92(3):227-37, Medical Imaging and Medical Informatics (MIMI) 